\title{
SOZIALPÄDAGOGISCHE BEGLEITUNG VON JUNGEN ERWACHSENEN IM INTERNATIONALEN FREIWILLIGENDIENST: Inbetweenness als Reflexionsmöglichkeit
}

\author{
Dr. Katharina Mangold \\ Wissenschaftliche Mitarbeiterin am Institut für Sozial- und Organisationspädagogik \\ Universität Hildesheim | katharina.mangold@uni-hildesheim.de
}

\begin{abstract}
Immer mehr junge Erwachsene in Deutschland nutzen die Möglichkeit zwischen Schulabschluss und Berufsausbildung bzw. Hochschulbildung, um Erfahrungen im Ausland zu sammeln und engagieren sich freiwillig in sozialen Projekten und Einrichtungen. Eine Möglichkeit dies zu tun stellt der entwicklungspolitische Freiwilligendienst weltwärts dar. Jungen Erwachsenen im internationalen Freiwilligendienst wird zugeschrieben, wichtige und prägende Erfahrungen zu machen (Mundorf 2000; Hudson/Inkson 2006). Dabei bergen Auslandsaufenthalte immer auch die Gefahr stereotypes Wissen zu verfestigen und zu (re)produzieren (Mangold 2013; Simpson 2004). In diesem Zusammenhang kommt der sozialpädagogischen Begleitung ein zentraler Stellenwert im internationalen Freiwilligendienst zu. Insbesondere Reflexionsmöglichkeiten während des Freiwilligendienstes werden für die Begleitung vorgeschlagen, da in der ethnographischen Studie, auf welcher der Beitrag basiert, deutlich gemacht wird, dass die jungen Menschen sich in Ambivalenz-Situationen bewegen, in denen sie nach angemessenen Verhaltensformen suchen. So kann an bereits vorhandene Potentiale angeknüpft und die sozialpädagogische Begleitung der Freiwilligen weiterentwickelt werden.
\end{abstract}

Schlagwörter: Internationaler Freiwilligendienst, sozialpädagogische Begleitung, Stereotype, junge Erwachsene, Reflexion und Diversity

The social pedagogical mentoring of young adults doing international volunteering inbetweenness as an opportunity for reflection

Increasing numbers of young people in Germany are taking up the opportunity to gain experience abroad during a gap year between finishing school and starting university or vocational training, by volunteering with social projects and institutions. One way of doing this, for example, is through the development aid volunteering service "weltwärts". Young adults doing international volunteering are said to gain important, formative experience (Mundorf 2000; Hudson \& Inkson 2006). However, spending time abroad always also runs the risk of reinforcing, producing or reproducing stereotypes (Mangold 2013; Simpson 2004). In thiscontext, social pedagogical mentoring plays a central role for international volunteers. One particular element proposed for the mentoring is to provide opportunities for reflection, as the ethnographic study on which this article is based shows that the young people move about in situations of ambivalence, in which they search for suitable forms of behaviour. Thus, social pedagogues can pick up on pre-existing potential and the volunteers 'social pedagogical mentoring can be extended.

Keywords: international volunteering, social pedagogical mentoring, stereotype, young adult, reflection and diversity 


\section{Einleitung}

Immer mehr junge Menschen aus westlichen Gesellschaften nutzen verschiedene Möglichkeiten mehrere Wochen oder Monate im Ausland zu leben (Mau 2007), sodass von einer „Normalisierung“ von Auslandsaufenthalten für die bildungsnahe Mittelschicht gesprochen werden kann: „Auslandsaufenthalte während des Studiums gehören inzwischen zu den vielen normativen Anforderungen, die eine Biografie für eine erfolgreiche berufliche Karriere mitbringen sollte“ (Bührmann/ Rehaag 2009: 35). Auch ein internationaler Freiwilligendienst - wie beispielsweise über das Programm weltwärts ${ }^{1}$ gefördert - stellt solch eine Möglichkeit für junge Menschen dar.

Trotz kritischer Stimmen und kontroverser Diskussionen (z. B. Töpfl 2008), ist ein Auslandsaufenthalt für die jungen Menschen selbst meist eine wichtige Erfahrung und findet zweifelsohne in einer prägenden Lebensphase statt, welche als Übergang verstanden wird (Stauber/Walther 2002). Diese Übergangsphase ist durch vielfältige Entgrenzungsprozesse gekennzeichnet - Selbstverständlichkeiten und Sicherheiten lösen sich auf (Beck 1986) und junge Erwachsene bewegen sich in verschiedenen Suchprozessen: Veränderung von Freundschaften, Ablösung vom Elternhaus, Entwicklung von Lebensperspektiven, Identitätssuche.

Programme wie weltwärts adressieren junge Menschen in dieser Übergangsphase. Die Frage nach der sozialpädagogischen Begleitung impliziert notwendigerweise auch die Frage nach der Zielgruppe des Programms. Die bisherige Praxis der Auswahl von Freiwilligen muss sich kritisch fragen lassen, wem die Erfahrung eines Auslandsaufenthaltes ermöglicht wird, wer sich diese leisten kann und wem sie verwehrt bleibt (Steinkühler 2014). Werden - wie bisher von den meisten Anbietern internationaler Freiwilligendienste - insbesondere Abiturient_innen erreicht (Jones 2005; Stern u. a. 2011), so stellen sich andere Anforderungen an die Begleitung, wenn auch junge Menschen mit anderen Bildungshintergründen oder junge Menschen in herausfordernden Lebenssituationen erreicht werden sollen. Die Schwierigkeit möglichst „gefestigte“ junge Menschen ins Ausland zu senden, macht eine Selektion der jungen Erwachsenen erforderlich, die nicht mehr den Prinzipien eines „Lerndienstes (für alle)“ entsprechen. Verträglichkeit und Kooperationsbereitschaft, Offenheit und Lernbereitschaft, Widerstandfähigkeit

1 Weltwärts ist ein Förderprogramm des Bundesministerium für wirtschaftliche Zusammenarbeit und Entwicklung (BMZ), das 2007 aufgelegt wurde, um 18- bis 28jährige jungen Menschen aus Deutschland einen Freiwilligendienst in sogenannten Entwicklungsländern für 6 bis 24 Monaten zu ermöglichen. Ich beziehe mich in diesem Artikel beispielhaft auf das Programm weltwärts. Dies lässt sich zweifach begründen: Erstens waren die jungen Menschen, die ich in Uganda beobachten konnte, fast alle über das Programm weltwärts gefördert; zweitens sind die konzeptionellen Überlegungen im Rahmen des weltwärts-Programms zur pädagogischen Begleitung recht fortgeschritten (GIZ 2014) und dennoch lassen sich daran meines Erachtens mit den empirischen Ergebnissen meiner Untersuchung weitere Verbesserungen vornehmen. Dabei muss darauf hingewiesen werden, dass die Datenerhebung meiner Untersuchung bereits vier Jahre zurückliegt und die Konzepte zur Begleitung sich kontinuierlich weiterentwickeln. 
und Reflexionsvermögen werden als Auswahlkriterien von Freiwilligen benannt (GIZ 2014), was aber möglicherweise viele junge Menschen erst in einem solchen Auslandsaufenthalt lernen würden. Meines Erachtens sind junge Menschen qua Lebensphase nicht „gefestigt“, sie sind auf der Suche, sie probieren sich aus (s. o.; Mangold 2011). Die Frage ist nun, wie ein sozialpädagogisches Begleitprogramm aussehen kann, um möglichst vielen jungen Menschen Erfahrungen im Ausland zu ermöglichen. Dann misst sich der „Erfolg“ des Freiwilligendienstes nicht mehr daran, ob ein Projekt aufgebaut wurde oder besonders gut gearbeitet wurde, sondern daran, ob ein junger Mensch etwas für sich gelernt hat und sich weiterentwickeln konnte. Ob dazu der Kontext der Entwicklungszusammenarbeit als Feld angebracht ist, kann und soll hier nicht beantwortet werden und würde in ein anderes Diskussionsfeld führen.

Daher spreche ich im vorliegenden Artikel von „sozialpädagogischer“ Begleitung und möchte damit ausdrücken, dass junge Menschen in der Übergangsphase, in welcher sie sich biographisch befinden, individuell unterstützt und begleitet werden müssen. Möchte man „nur“ jungen Menschen die Möglichkeit eines Freiwilligendienstes geben, die angeblich mit beiden Beinen im Leben stehen und gefestigt sind, dann verlangt diese eine andere (möglicherweise weniger aufwändige) Begleitung, als wenn man jungen Menschen die Möglichkeit eines Freiwilligendienstes eröffnet, die sich in schwierigen Lebenssituationen befinden. Eine sozialpädagogische Begleitung - verstanden als individuelle, bedarfsgerechte Unterstützung ermöglicht es meines Erachtens, auch junge Menschen zu unterstützen, die sich bisher in Bewerbungsverfahren um einen Platz in einem internationalen Freiwilligendienst nicht durchsetzen können, weil ihnen Krisen und Probleme zugeschrieben werden, die sie alleine im Ausland nicht bewältigen können. Dieser Exkurs zur Frage der Zielgruppenbestimmung kann im Weiteren nicht vertieft werden, da die empirischen Daten, welche diesem Artikel zugrunde liegen, stets mit bildungsorientierten und sozial integrierten jungen Menschen zu tun haben. Dennoch wird in Kapitel 4 des vorliegenden Artikels deutlich, dass sich auch die Zielgruppe der Abiturient_innen in ganz verschiedenen Lebenskontexten befinden und verschiedene biographische Krisen während des Freiwilligendienstes im Ausland bearbeitet werden (müssen). Die von mir beobachteten Freiwilligen in Uganda erhielten zumeist eine Begleitung im Sinne von Vorbereitungsseminar, Zwischenseminar und Rückkehrseminar im Umfang von insgesamt 25 vorgeschriebenen Seminartagen im Programm weltwärts. Dies reicht jedoch nicht aus, sondern es bedarf einer intensiveren Begleitung vor Ort, um der Gefahr der Stereotypen(re)produktion entgegenzuwirken. Aktuelle Überlegungen zur Begleitung von Freiwilligen wie sie in der pädagogischen Dokumentation der GIZ (2014) zu entnehmen sind, machen auf eine weitreichende Weiterentwicklung aufmerksam, in der auch Ansprechpersonen vor Ort und Peer-learning angeregt und Berichte als Reflexionsinstrument institutionalisiert werden. 
Ich möchte mit diesem Artikel der Frage nachgehen, die mir in den vergangenen Jahren von Freiwilligen selbst und vor allem von Entsendeorganisationen immer wieder gestellt wurde: Wie kann eine Begleitung von jungen Menschen in einem internationalen Freiwilligendienst gut gelingen? Wie kann Begleitung aussehen, welche Strukturen sind erforderlich und welche Inhalte sind wichtig? Ich kann dazu kein Rezept anbieten - nicht nur, weil es mir meine Beobachtungen und Erfahrungen nicht möglich machen, sondern auch, weil ein fertiges Rezept dem „sozialpädagogischen Können“ (Müller 2006), das auch in diesem Feld und insbesondere in der Begleitung der jungen Menschen erforderlich ist, entgegenstehen würde. Und hiermit ist wohl schon eine erste Prämisse verraten: Es bedarf meines Erachtens einer sozialpädagogische Begleitung von jungen Menschen im internationalen Freiwilligendienst und damit verbunden eines individuellen Ansatzes der Begleitung. Dennoch lassen sich strukturelle Überlegungen und inhaltliche Aspekte ableiten, die für und in der sozialpädagogischen Begleitung von Freiwilligen relevant sind. Dazu soll dieser Artikel einen Beitrag leisten und zur Diskussion anregen.

Im Folgenden werde ich in vier Schritten vorgehen: Zunächst wird kurz das methodische Vorgehen der ethnographischen Studie vorgestellt, auf deren Ergebnisse in diesem Artikel zurückgegriffen wird. Als zentrales Analyseergebnis lässt sich festhalten, dass die Freiwillige in Ambivalenzen agieren (müssen) und häufig nicht wissen, wie sie sich „richtig“verhalten sollen. Diese Ambivalenzsituationen bezeichne ich als Inbetweenness, welches somit zentrales Moment des Freiwilligendienstes darstellt. Drittens werde ich fragen, was sich anhand dieser Analyse und der Situation des Inbetweenness für die sozialpädagogische Begleitung der Freiwilligen ableiten lässt. Abschließend werden die Überlegungen in einem Fazit gebündelt, mit dem Ziel einige Denkanstöße für die sozialpädagogische Begleitung im internationalen Freiwilligendienst zu geben.

\section{Methodisches Vorgehen}

Der Beitrag basiert auf einer ethnographischen Studie, die nach Erfahrungskonstruktionen junger Freiwilliger aus Deutschland im Einsatzland Uganda fragt und von mir zwischen 2008 und 2012 durchgeführt wurde. Innerhalb einer dreimonatigen Erhebungsphase von Oktober 2009 bis Januar 2010 wurden junge Freiwillige aus Deutschland in ihrem Alltag in Uganda beobachtet. Die Kontaktaufnahme mit den jungen Erwachsenen erfolgte vor dem Antritt ihres Freiwilligendienstes per E-Mail. Bei der Auswahl der jungen Erwachsenen wurde - gemäß des „theoretical samplings“ (Glaser/Strauss 1998) - auf vielfältige Kontraste geachtet: Geschlecht, Einsatzbereiche, Stadt-Land, informell im Ausland oder mit einer Organisation (die meisten jungen Menschen wurden durch das Programm weltwärts unterstützt). Zum Zeitpunkt der Teilnehmenden Beobachtungen waren die jungen Menschen bereits zwischen 3 und 5 Monaten in Uganda und hatten die erste Phase 
des Ankommens meist hinter sich. Mit den fünf kontaktierten Freiwilligen fanden jeweils ca. zehn Beobachtungstage statt, während derer ich die jungen Menschen in ihrem Alltag begleitete (ich wohnte meist bei den Freiwilligen, ging mit ihnen zur Arbeit, aber auch das Leben abseits der Arbeit wie Einkaufen, Freund_innen treffen usw. war Teil der Beobachtung). Insgesamt wurden 22 Freiwillige Teil der Beobachtungen, die in 44 Beobachtungsprotokollen mit etwa 220 Seiten Umfang festgehalten wurden. Die Protokolle wurden kodiert und die thematischen Felder sequenzanalytisch ausgewertet.

Durch die Beobachtung des Alltagslebens der Freiwilligen konnten Erkenntnisse über die von den Freiwilligen erlebten Herausforderungen und deren Bearbeitung gewonnen werden. Hier schließt das Thema der Reflexion und damit verbunden der Frage nach der sozialpädagogischen Begleitung dieser Prozesse an. Dabei kann es im Folgenden keine eindeutige Verortung zwischen „Ohne pädagogische Begleitung kein Lernen“ und „Freiwilligendienste sind überpädagogisiert“ geben, sondern es wird auf Möglichkeiten und Grenzen sozialpädagogischer Begleitung aufmerksam gemacht und anhand des empirischen Materials herausgearbeitet, welche Herausforderungen die jungen Menschen erleben und welche Formen und Inhalte einer sozialpädagogischen Begleitung notwendig bzw. möglich sind. So wird nun im dritten Kapitel das Ergebnis der empirischen Analyse anhand der zentralen Kategorie Inbetweenness dargestellt, um daran anschließend in Kapitel 4 auf die Frage der sozialpädagogischen Begleitung näher einzugehen.

\section{Inbetweenness als zentrales Moment des Freiwilligendienstes}

Unter Inbetweenness wird in der empirischen Analyse der Daten eine Art „Dazwischen-Sein" verstanden, in welchem sich die Freiwilligen bewegen. Der Freiwilligendienst ist gekennzeichnet durch eine Art Auszeit und Ernstcharakter zugleich - die Freiwilligen engagieren sich für andere und für sich selbst, sie reproduzieren Vertrautes und stellen zugleich neue Normalitäten her. Sie konstruieren Differenzen zwischen schwarz und weiß und hier und dort. Dieses Inbetweenness eröffnet den Freiwilligen jedoch die Möglichkeit, sich nicht sofort entscheiden zu müssen und bietet somit eine Reflexionsmöglichkeit (Mangold 2013).

Dennoch sind die jungen Menschen herausgefordert, sich zu verorten und ihre Position zu finden. Es kann herausgearbeitet werden, dass die jungen Menschen um adäquates Verhalten in den jeweiligen Situationen bemüht sind und ihr Handeln von der Suche nach angemessen Verhalten geprägt ist.

Als Beispiel kann hier ein junger Mann namens Michael $^{2}$ angeführt werden, der nach seinem Hochschulstudium der Sozialen Arbeit einen Freiwilligendienst über

2 Alle Namen der Freiwilligen im Sample wurden geändert. 
weltwärts in Uganda absolviert. Er unterhält sich mit der Beobachterin über zwei ugandische Männer in seinem Garten, die seinen Rasen mit einer Machete mähen. Michael ist darum bemüht, sich angemessen zu verhalten, fühlt sich aber dennoch „komisch“, wie im folgenden Auszug aus dem Beobachtungsprotokoll deutlich wird:

„Leute, die seinen Garten richten, das sei schon auch komisch, so seierja eigentlich gar nicht, aber hier kann man es sich halt leisten und wäre alleine auch über zwei Tage beschäftigt, den eigenen Rasen zu mähen." (BP 36, Z. 79-81)

Michael berichtet davon, dass ugandische Männer „seinen“ Rasen mähen und begründet es damit, dass er alleine zwei Tage damit beschäftigt wäre. Aber ganz wohl ist ihm dabei nicht und er artikuliert „es sei schon auch komisch“. Es stellt sich die Frage, was hinter dem „auch" steckt, ob er damit zum Ausdruck bringen möchte, dass „auch“ andere Dinge in Uganda komisch seien, dass Uganda generell komisch sei bzw. ob sein Verhalten in Uganda komisch sei. Gleichzeitig distanziert er sich von seinem Handeln bzw. distanziert sein aktuelles Handeln von seinen Charakterzügen, weil „so sei er ja eigentlich gar nicht“. Er verhält sich also in Uganda aktuell so, wie er „eigentlich“ gar nicht ist, aber hinter dem „eigentlich“ steckt dennoch ein Wesenszug von ihm. Er nimmt weiterhin Bezug auf die Bedingungen, indem er einerseits argumentiert, dass er alleine zwei Tage dafür benötigen würde, um den Rasen selbst zu mähen und andererseits, dass die Arbeitskräfte in Uganda so günstig sind, dass ,man“ - bzw. im konkreten Fall er - sich das leisten kann. Er differenziert zwischen den Bedingungen in Deutschland - dies wird zwar nicht explizit angesprochen, doch in dem „hier" wird gleichzeitig auch ein „dort" hergestellt und sozusagen ein Kontrast aufgemacht, welcher Deutschland sein muss, wo man es sich nicht leisten kann - und den Bedingungen in Uganda. Und da er in Uganda andere Bedingungen vorfindet, handelt er auch anders. Dabei fühlt er sich „schon auch komisch“.

Aber es scheint keine Alternative zu geben - ein „richtiges“ ${ }^{3}$ Handeln ist nicht möglich, darauf macht auch ein anderer Freiwilliger - der 20jährige Jakob - in einer kurzen Erzählung aufmerksam. Jakob distanziert sich von der Praxis des „going native“, wie er es bei anderen Freiwilligen oder anderen Europäer_innen erlebt und was er als Anpassung an das ugandische Verhalten interpretiert. Dazu berichtet er von einer Finnin, die ugandische Kleidung trägt und die Sprache spricht, aber dennoch nicht ernst genommen wird und von einem ugandischen Mann nachgeäfft wird. Dies verleitet Jakob zu der Aussage: „Man könne also mit dem So-SeinWollen wie die Einheimischen auch nichts erreichen, das sei eher noch schwieriger".

3 "Richtig“ darf hier nicht als normative Idee verstanden werden, die an die jeweiligen Situationen angelegt wird und das Handeln der Freiwilligen bewerten soll, sondern "richtig“ muss als subjektive Konstruktion der Freiwilligen selbst gesehen werden und damit kommt zum Ausdruck, dass sie letztlich für ein gutes und richtiges Handeln keinen Ausweg sehen. 
Mit dieser Äußerung macht er deutlich, dass es nicht um Anpassung oder Ablehnung gehen kann und es keine eindeutige Antwort gibt. Er agiert in Situationen, die nicht nur durch hohe Komplexität (sprachliche Missverständnisse), sondern durch Ambivalenzen, d.h. nicht auflösbare Dilemmata gekennzeichnet sind. Alles ist schwierig, „das So-Sein-Wollen wie die Einheimischen [ist] noch schwieriger" aber alles andere - das nicht-so-sein-wollen - eben auch. Es gibt kein einfaches Verhalten. Der Umgang mit Differenzen kann nicht produktiv sein, er sieht sozusagen keinen Ausweg.

Dennoch sind die Freiwilligen in den konkreten Handlungssituationen herausgefordert, sich kontinuierlich zu verorten. Dabei lässt sich im Datenmaterial die Tendenz erkennen, Differenzen zu konstruieren, die dem Muster des entweder-oder zuzuordnen sind. Die komplexen Handlungssituationen werden aufgelöst, indem Klarheit hergestellt wird und ein „hier“ und „dort“ die Sachlage erklärt. Somit wird nach klaren Handlungsanweisungen oder klaren Prinzipien gehandelt und es müssen nicht ständig neue Entscheidungen getroffen werden. Diese Strategie kann an folgendem Auszug des Beobachtungsprotokolls erläutert werden: Jakob berichtet, wie er von einem kranken Mann angebettelt wurde.

„Jakob erzählt Markus von dem Mann, der sie neulich angebettelt hat. Den Mann kennen sie aus dem parish [Kirchengemeinde]. Er hatte einen Bodaunfall [Unfall mit dem Mofa-Taxi] und eine Verletzung an der Hand, weshalb er in Mubende im Krankenhaus war. Er musste für die Medikamente so viel Geld ausgeben, dass er nichts mehr hatte, dann hat er Jakob und Markus angebettelt. Aber sie haben beschlossen da nichts zu geben, das sei einfach ein Fass ohne Boden, dann kommt der nächste und hat Schmerzen und braucht das und das. Es ist einfach schwierig. Man muss denen auch vermitteln, dass man selbst Schüler ist und auch nicht so viel hat, sagt Jakob. "(BP 12, Zeile 447-452).

Jakob setzt klare Grenzen. Das Betteln steht im konkreten Kontext einer schwierigen Lebenssituation des Mannes, da er „einen Bodaunfall“ hatte und „im Krankenhaus war". Jakob und Markus reagieren aber nicht auf eine Ausnahmesituation, obwohl sie den Kontext kennen und wissen, dass der Mann im Krankenhaus Geld für die Medikamente zahlen musste und jetzt nichts mehr hat. „Nichts mehr“ bedeutet hier wohl, dass er sich weder Transport noch Nahrungsmittel leisten kann, da er im Krankenhaus bereits das Geld ausgegeben hat. Sie wissen um die schwierige Situation, aber sie handeln standardisiert und zwar nach dem Prinzip „nichts zu geben“. Dass sie aber etwas „beschlossen“ haben, weist auf eine Diskussion oder eine Auseinandersetzung über dieses Thema hin, die jedoch abgeschlossen ist. Das Prinzip ist beschlossene Sache. Denn anscheinend haben Jakob und Markus hier eine klare Antwort, sie kommen nicht ins Grübeln, werden nicht wackelig. Das Prinzip wird nicht in jeder neuen Situation des Angebettelt-Werdens umgeworfen, es gibt keine Ausnahmen, auch nicht bei Menschen, die man kennt 
und die in Notlagen geraten sind. Mit dieser klaren Verortung müssen sie sich nicht jedes Mal aufs Neue mit der Frage auseinandersetzen „Was nun?“. Das Handeln nach klaren Prinzipien verleiht somit Handlungssicherheit.

Der Aspekt der Verallgemeinerung wird in der Metapher „Fass ohne Boden“ deutlich. Es ist sozusagen aussichtslos, es würde kein Ende nehmen und so erzeugen sie eine scheinbare Gerechtigkeit, indem sie alle gleich behandeln, indem sie sich diesem Fass entziehen und klar Position beziehen. Weil sonst „kommt der nächste“, es gibt so viele Schwierigkeiten und so viele Menschen in Notlagen, es wird kein Ende nehmen und der nächste „braucht das und das“. Durch diese Art „Gleichbehandlung“ - niemanden etwas zu geben - müssen sie ihr Verhalten nicht ständig rechtfertigen.

In dem Satz „es ist einfach schwierig“ drückt sich erneut eine Ambivalenz aus. Jakob ist nicht zufrieden mit der generellen Entscheidung nichts zu geben. Er ist nicht überzeugt davon, dass es der richtige Weg ist, aber er weiß, dass etwas zu geben für ihn keine Lösung ist. Zum Abschluss der Szene nimmt er Bezug auf seine eigene Lebenssituation des Schüler-Seins und verbindet damit die Aussage, dass auch er nicht viel Geld hat. Die Frage danach was „viel“ ist, bleibt jedoch offen. Wichtig scheint es Jakob aber, dies den Ugander_innen zu vermitteln. Er will nicht als jemand dastehen, der einfach nichts gibt, der nicht helfen will oder dem die Sorgen der Menschen egal sind. Sondern er möchte verstanden werden, dass er nicht bedingungslos helfen kann und seine Mittel begrenzt sind. Er will also damit ausdrücken, dass er zwar reicher als die Ugander_innen ist, aber im Grund auch nichts hat. Somit erzeugt er in einer Situation, die auf Differenz aufbaut, Gleichheit bzw. minimiert die Differenz - denn im Grunde haben sie beide „nicht viel“. Hier wird eine Abstufung der Differenz deutlich.

Durch die Herstellung von Differenzen (im Sinne von entweder-oder) verorten sich die Freiwilligen und stellen Klarheit her. In ihrem Denken und Handeln orientieren sie sich kontinuierlich zwischen den beiden Orientierungsrahmen Uganda und Deutschland. Es wird dabei ein „hier“ und ein „dort“ hergestellt, auch wenn dies nicht immer explizit erwähnt wird. Gleichzeitig muss auch erwähnt werden, dass es Situationen gibt, in denen weniger Differenzen im Fokus stehen, sondern vielmehr ein sowohl-als-auch konstruiert wird. Hier werden neue Perspektiven entwickelt und nicht das Eine gegen das Andere gestellt.

Zusammenfassend kann festgehalten werden, dass die zentrale Herausforderung für die jungen Menschen im internationalen Freiwilligendienst das „Ringen um angemessenes Verhalten“ darstellt und dass sie häufig nicht wissen, was „richtig“ ist. Bevor jedoch das Handeln über klare Prinzipien im Schema entweder-oder in eine Verfestigung eine bipolaren Struktur führt, befinden die Freiwilligen sich im sogenannten Inbetweenness - einer ambivalenten Situation, die gerade aufgrund ihrer Unsicherheit und des Nicht-Wissens-Wie Chancen zur Reflexion und damit 
verbunden zur Bildung bietet. Dieses Inbetweenness kann somit als Grundstruktur des Freiwilligendienstes verstanden werden. Im Folgenden wird danach gefragt, wie Freiwillige, die sich immer wieder in diesen ambivalenten Situationen befinden, in ihrer Auseinandersetzung begleitet werden können.

\section{Freiwillige begleiten - vorhandene Potentiale nutzen}

Wie bereits zu Beginn des Artikels zum Thema Zielgruppenbestimmung erläutert, kann nach wie vor von einer eher homogenen Freiwilligengruppe ausgegangen werden, so auch in der von mir durchgeführten ethnographischen Studie. Würde also bereits im Auswahlprozess zum Freiwilligendienst eine höhere Heterogenität der Freiwilligen angestrebt, würden sich die Unterstützungsbedarfe möglicherweise nochmals vervielfältigen. Alle jungen Menschen im Rahmen der Studie befinden sich zwischen Abitur und Studium oder zwischen Studienende und Berufseinstieg, arbeiten zwischen 6 und 12 Monaten in sozialen Projekten in Uganda, sind zwischen 18 und 26 Jahre alt und waren meist schon vor ihrem Freiwilligendiensten im sozialen Bereich engagiert. Trotz dieser ähnlichen Lebenssituationen sind die jungen Menschen mit ganz unterschiedlichen Herausforderungen in ihrem Freiwilligendienst im Ausland konfrontiert und müssen ihren Umgang damit finden. Exemplarisch dazu werden nun fünf Freiwillige vorgestellt: (1) Dominiks Leben in Uganda ist geprägt von Einsamkeit und er findet wenig Kontakt zu Ugander_ innen. Er ist in regelmäßigem Telefonkontakt mit seiner Mutter. Während einer Zwischenkrise sucht er Kontakt zur Entsendeorganisation und bekommt auch Gesprächsangebote. Was ihm jedoch hilft sind die informellen Kontakte zu anderen Freiwilligen und das Gefühl, nicht mehr alleine zu sein. (2) Carmen ist hingegen in Uganda gut eingebunden, pflegt Kontakt zu ihrem Freund in Deutschland über Skype und hat auch in Uganda viele neue Bekannte gefunden. Beim Arbeiten ist sie sehr engagiert und genießt es, eigene Gestaltungsfreiräume zu haben und gebraucht zu werden. (3) Maria will sich engagieren, wird jedoch von ihrer Aufnahmeorganisation nicht gebraucht. Sie lebt weit außerhalb der Stadt und ist viel alleine mit den Mitarbeiter_innen der Einrichtung. An den Wochenenden trifft sie sich mit einer Freiwilligen, die sie auf dem Zwischenseminar kennengelernt hat und hat viele ugandische Freund_innen in Kampala, wo sie regelmäßig hinfährt. Ereignisse, denen sie beiwohnt, wie dem Selbstmordversuch eines Angestellten, führen letztlich dazu, dass sie ihren Freiwilligendienst nach acht Monaten beendet. Die Entsendeorganisation entschuldigt sich für die nicht vorhandene Arbeitsstruktur vor Ort und bietet ihr ein Reflexionsgespräch an. Während der Schwierigkeiten vor Ort gab es vereinzelte Telefonate, dennoch fühlt sich Maria wenig unterstützt. (4) Auch Lena ist vor die Herausforderung gestellt sich ihre eigene Arbeit zu strukturieren und Aufgaben zu suchen. Dabei hat sie in der Zeit des Freiwilligendienstes den Todesfall ihres Vaters zu verarbeiten. Sie besucht ihn nach der tödlichen Diagnose in Deutschland und fliegt ein zweites Mal während ihres 
Freiwilligendienstes zur Beerdigung nach Deutschland. Die Entsendeorganisation steht ihr dabei einerseits begleitend zur Seite, rückt dann aber formale Fragen wie Urlaubstage in den Vordergrund. (5) Jakob hingegen verbringt eine unbeschwerte Zeit in Uganda, reist viel und genießt es, sich mit anderen Freiwilligen zu treffen und muss keine offensichtlichen Herausforderungen meistern.

Ich möchte im Folgenden weniger die konkreten Einzelfälle der Freiwilligen betrachten, sondern vielmehr anhand der erhobenen Daten fragen, auf was in der Begleitung der Freiwilligen zurückgegriffen werden könnte - also Potentiale sichtbar machen, die meines Erachtens bereits vorhanden sind. Dass die Begleitung dabei stets an individuelle Biographien rückgebunden werden muss, macht die kurze Vorstellung der verschiedenen Freiwilligen deutlich. Zwar arbeiten alle in sozialen Einrichtungen in Uganda und werden von einer Organisation unterstützt, dennoch sind die jeweiligen Lebenssituationen verschieden. Während Dominik eher mit Einsamkeit zu kämpfen hat, blüht Jakob im Partyleben auf. Carmen hingegen will über ihre Arbeit etwas erreichen, während Maria spürt, dass sie nicht erwünscht ist. Lena muss sich mit dem Tod ihres Vaters auseinandersetzen. All diese individuellen Geschichten wollen und müssen begleitet werden, was die Notwendigkeit einer sozialpädagogischen Unterstützung von jungen Erwachsenen im internationalen Freiwilligendienst deutlich macht.

Meines Erachtens lassen sich vier zentrale Aspekte herausarbeiten, welche Hinweise für die Begleitung von jungen Menschen im internationalen Freiwilligendienst liefern können, diese werden im Folgenden vorgestellt.

\subsection{Freiwilligendienst ist mehr als Arbeit: den Alltag begleiten}

Wie im Fall von Maria deutlich wurde, ist es zunächst notwendig, eine geeignete Einsatzstelle zu finden und hierbei auch die Kommunikation zwischen der Entsendeorganisation aus Deutschland und der Aufnahmeorganisation aus Uganda zu gewährleisten. Es braucht klare Ansprechpersonen und darf nicht nur auf die informellen Kontakte mit ehemaligen Freiwilligen beschränkt sein. Die Partnerorganisation (oder Aufnahmeorganisation) muss als Kooperationspartner agieren und auch Kompetenzen der Begleitung der jungen Menschen übertragen bekommen. Eine Einrichtung, mit der zusammengearbeitet wird, ist in den allermeisten Fällen vorhanden, auch wenn von einer Kooperation auf „Augenhöhe“ - wie es so schön heißt - noch lange nicht gesprochen werden kann. ${ }^{4}$

4 In diesem Kontext kann auf Forschungen hingewiesen werden, die sich mit dem Nutzen eines Internationalen Freiwilligendienst für die Empfängerorganisationen im globalen Süden beschäftigen (bspw. Schwinge 2011; Klocke 2013; Haas 2012; Lough 2013). Hierbei wird deutlich, dass insbesondere eine Erweiterung der Netzwerke und politische Öffentlichkeit für die Aufnahmeorganisation von zentraler Bedeutung sind, was meines Erachtens nicht auf ein Miteinander auf Augenhöhe schließen lässt. 
Darüber hinaus muss berücksichtigt werden, dass es bei der Begleitung des internationalen Freiwilligendienstes nicht nur um die Begleitung und Reflexion der Tätigkeit im Rahmen der Arbeitsstelle geht: Ein Freiwilligendienst im Ausland besteht aus mehr als Arbeit. Das mag zunächst „bevormundend“ klingen, könnte man doch auch davon ausgehen, dass die jungen Menschen erwachsen sind und ihr Sozialleben selbständig und eigenverantwortlich organisieren. ${ }^{5}$ Ich möchte nicht dafür plädieren, den jungen Menschen diese Kompetenz abzusprechen. Jedoch muss auch klar sein, dass Erfahrungen, welche die Freiwilligen in ihrem Freiwilligenjahr machen, nur zu einem - und wie die empirischen Daten zeigen - zu einem relativ kleinen Teil im Bereich der Einsatzstelle und somit der Arbeit selbst stattfinden. Themen wie Armut, Freundschaften, kulturelle Differenzen oder Statusfragen beschäftigen die jungen Menschen in ihrem Alltag mindestens ebenso und müssen darum reflektiert werden. Auf dem Weg zur Arbeit werden sie auf der Straße um Essen und Geld gebeten, in ihrer Wohnung müssen sie einen Weg finden mit der Reinigungskraft umzugehen, in der Kneipe müssen sie entscheiden, mit wem sie ein Bier trinken und mit wem nicht. Hinzu kommt, dass die jungen Menschen viele Situationen das erste Mal erleben - das erste Mal in einer 40-Stunden-Arbeitswochen, häufig das erste Mal für längere Zeit weg von Zuhause, meist das erste Mal in Uganda.

Diese verschiedenen Erfahrungen das erste Mal zu machen und dabei im Ausland zu sein, deutet auf eine Doppelstruktur hin und so lässt sich von einer Intensivierung der Orientierungsphase sprechen: die jungen Menschen agieren mit Herausforderungen zwischen Jugendlich-Sein und Erwachsen-Sein und mit den Herausforderungen, die der Ortswechsel und der neue Kontext Uganda für sie darstellt. Fürstenau und Niedrig (2007) lehnen sich an King (2005) an und sprechen von Komplexitätszugewinn in der Jugendphase, wenn diese im Migrationskontext geschieht. Günther (2009) geht einen Schritt weiter und spricht von „doppelter Krise“. Auch wenn meines Erachtens vor allem der Begriff der Krise hier in die Irre führt, da er die Lesart einer Problematisierung und damit verbunden eine mögliche Stigmatisierung nahe legt, so schließe ich mich dahingehend den Autorinnen an, als dass ich von einer Intensivierung der Orientierungsphase spreche, wenn junge Menschen im Ausland agieren. In einer Phase, in der sich die jungen Menschen selbst suchen, findet ein Ortswechsel statt, der neue Herausforderungen beinhaltet, da sie mit weiterem „Unbekannten“ - zu dem „Neuen“ der jungen Erwachsenenphase - umgehen müssen. Dabei sind die Herausforderungen am neuen Ort und die Herausforderungen der Jugendphase nicht in Konkurrenz zueinander zu verstehen, sondern sie ergänzen und intensivieren sich. Wichtig für die Begleitung von jungen Menschen erscheint mir dabei insbesondere, zu erkennen, dass es nicht

5 So macht auch die GIZ (2014) deutlich, dass die Begleitung die Lebensphase mitberücksichtigen muss, ohne dabei durch Überbehütung eigene Lernprozesse zu verhindern. 
nur um die Reflexion der Arbeitssituation geht, sondern der Internationale Freiwilligendienst in einem größeren Zusammenhang steht und vor allem auch Alltagserfahrungen verarbeitet und begleitet werden müssen. Außerdem wird darin deutlich, dass nicht nur die „Auslandssituation“ als Folie der Reflexion herangezogen werden darf, sondern auch die „Jugendphase“ als strukturierend für die Erfahrungen und Erlebnisse im internationalen Freiwilligendienst betrachtet werden muss.

\subsection{Diversity statt Interkulturalität: Offenheit für Neues}

Um die im vorangehenden Abschnitt beschriebene Reflexion zu ermöglichen, sollten weniger bipolare Konstruktionen wie sie die interkulturelle Pädagogik (zumindest implizit) nahelegt fokussiert werden, sondern hybride und vielfältige Deutungen im Sinne von Diversity aufgezeigt werden (Ansätze für die Begleitung der Freiwilligen dazu bspw. Goel 2011).

In den komplexen Handlungssituationen suchen die jungen Erwachsenen nach einfachen Handlungsmustern. Dass sie dabei auf stereotype Zuschreibungen zurückgreifen, verwundert nicht, da diese Stereotype wenig Platz für Komplexität lassen und somit einen klaren Orientierungsrahmen für die Freiwilligen bereithalten. Bührmann und Rehaag (2009) arbeiten in einem Projekt mit Studierenden aus Mexiko, China und Deutschland heraus, dass die jeweiligen Projektionen - also Stereotype - der Studierenden im Ausland, mit dem bisherigen Wissen über das Land des Auslandsstudiums verbunden ist. „Es scheint so zu sein, daß Erfahrungen leicht ein stereotypes Bild verfestigen, während Beweise des Gegenteils relativ wirkungslos bleiben. Ein Schwarzer mit einem Messer bestätigt das Vorurteil von der Gefährlichkeit schwarzer Männer. Hundert schwarze Männer ohne Messer können dieses Vorurteil nicht zerstören“ (Broek van der 1993: 64). So ist insbesondere darauf zu achten, diese vorhandenen Stereotype zu reflektieren, transparent zu machen und das größte Vorurteil zu durchbrechen, nämlich unser aller Vorurteilslosigkeit (Bührmann/ Rehaag 2009).

Inhaltlich lässt sich mit der Konstruktion der Bipolaritäten darauf hinweisen, dass die jungen Menschen sich ein Ordnungssystem schaffen, das bereits in ihrer Sozialisation angelegt ist. Möglicherweise wird das bipolare Denken in Vorbereitungsseminaren und Begleitseminaren über interkulturelle Trainings u. ä. weiter verstärkt, indem betont wird, wie Uganda ist, was zu erwarten ist und wie man sich verhalten soll. Je nachdem welches Bild von Uganda gezeichnet wird - junge Menschen in Slums oder junge Menschen an Hochschulen - entstehen verschiedene Bezugsrahmen. Hier ist auf die Untersuchung von Simpson (2004) zu verweisen, die deutlich macht, dass bereits das Werbematerial für einen internationalen Freiwilligendienst von bipolaren Konstruktionen durchzogen ist und dass die jungen Menschen im Freiwilligendienst essentialistische Konzepte von „den Anderen“ reproduzieren. Die bereits existierenden Bilder der Freiwilligen verfestigen 
sich während des Freiwilligendienstes häufig. So findet beispielsweise wenig Auseinandersetzung mit strukturellen Bedingungen bezüglich sozialer Ungleichheit statt, sondern die Lebensumstände werden über „Glück“ und „Pech“argumentiert (Simpson 2004).

So erzeugen bipolare Konstruktionen zwar Ordnung und Sicherheit, unterliegen jedoch der Gefahr, Stereotypen zu reproduzieren. Es ist meines Erachtens explizit Aufgabe der Begleitung von Freiwilligen mit diesen bipolaren Konstruktionen zu arbeiten, um einer Verfestigung von stereotypen Deutungen entgegenzuwirken und nicht - wie die Analyse von Simpson (2004) nahelegt - die bipolaren Konstruktionen zu unterstützen. Die Logik der Interkulturalität erzeugt möglicherweise aber eben ein bipolares Verständnis und trägt somit wenig dazu bei, Stereotype (beispielsweise bezüglich der Kultur) zu durchbrechen. So machen auch Leiprecht und Vogel (2008) darauf aufmerksam, dass trotz Bemühungen den Kulturbegriff zu dekonstruieren, sowohl im Alltagsverständnis, als auch in Politik und pädagogischer Praxis ein Kulturbegriff konstruiert wird, der Kultur mit Nationalstaat, Gesellschaft, Volk oder Nation gleichsetzt.

Diesbezüglich scheint für die Begleitung von Freiwilligen vor allem eine Bearbeitung der Ambivalenzsituation vor Ort sinnvoll zu sein, die zum Ziel hat, ambivalente Situationen nicht (immer) auflösen zu müssen, sondern sie als produktive Möglichkeitsräume der Reflexion und Auseinandersetzung zu nutzen (Mangold 2012b). Da sich junge Erwachsene hierbei ebenfalls qua Lebensphase in Ambivalenzsituationen befinden und diese bearbeiten, muss die Intensivierung der lebensphasenspezifischen und ortswechselbedingten Herausforderungen - von denen wie oben hervorgehoben ausgegangen werden kann - in der Begleitung mitberücksichtigt werden.

Wie Unterschiede hergestellt und dann auch bewertet werden, hängt von unserer eigenen Gewordenheit und Sozialisation ab (Bührmann/Rehaag 2009: 41). Für die pädagogische Begleitung bedeutet dies meines Erachtens auch einen Reflexionsprozess über das „Eigene“ und somit über sich selbst, die eigenen (normativen) Vorstellungen und eigenen Gewordenheiten anzuregen und anzuleiten. Damit verbunden ist ein Öffnungsprozess, der es möglich macht, die bisherigen Deutungen zu hinterfragen. Am Beispiel des Vorbereitungsseminars von Lena kann auf solche Öffnungsprozesse hingewiesen werden:

„Lena erzählt, dass für sie etwas ganz anderes wichtig gewesen ist und dass das in Deutschland bei dem Vorbereitungsseminar angefangen hat. Das Vorbereitungsseminar wurde von einem sehr, sehr reflektierten und tollen Menschen angeleitet und er ist für Lena inzwischen eine sehr, sehr wichtige Person, wie ein Vater, sagt sie am Schluss. Bei diesem Vorbereitungsseminar sei alles sehr, sehr intensiv gewesen, nach zwei Tagen war die Gruppe schon so 
eng zusammen, es war so eine Offenheit und am Ende gab es ein Reflexionsgespräch in zufällig ausgewählten Vierergruppen, wie man den anderen erlebt, wie man ihn in Veränderungen sieht und vieles mehr, und dieses Reflexionsgespräch hätte in ihrer Gruppe 14 Stunden gedauert. Da sei so viel angestoßen worden, so viel geöffnet worden, dass damit etwas ganz wichtiges anfing. Außerdem ging es darum, dass man nicht alleine und mit nichts nach Uganda geht, sondern ganz reich und gefüllt, weil alles Wichtige hat man in sich und das trägt man mit nach Uganda." (BP 39, 141-152)

Begrifflichkeiten wie „sehr, sehr intensiv“, „schon so eng “, „viel angestoßen“, „,so viel geöffnet" und „etwas ganz wichtiges anfing" weisen darauf hin, dass das Vorbereitungsseminar ein Einschnitt in Lenas Leben darstellt. An der Situation des Vorbereitungsseminars kann deutlich gemacht werden, dass sowohl die zentrale Figur des Seminarleiters - der von Lena wie ein Vater erlebt wird - als auch die Gruppe eine wesentliche Rolle spielen bei der Öffnung der Situation. Dies erinnert an einen Neustart und weist auf den Beginn einer einschneidigen Veränderung hin. Sie ist bereit für diese Veränderung bzw. das Vorbereitungsseminar hat dies ermöglicht, hat „so viel geöffnet“ und dann fing „etwas ganz wichtiges“ an. Das 14-stündige Reflexionsgespräch, das in einer zufällig gewählten Vierergruppe stattfindet, kann hier als zentraler Einschnitt verstanden werden. Wer sich 14 Stunden Zeit nimmt, um über sich selbst, wie die anderen auf einen wirken und wie man auf die anderen wirkt, zu sprechen, der setzt sich intensiv mit Fragen zur eigenen Person auseinander. Nicht in Uganda fängt etwas Neues an, sondern bereits in Deutschland beginnt das Neue und man könnte sagen, dass Uganda als Auslöser oder als Ermöglichung zu verstehen ist, etwas Neues anzufangen.

Das Vorbereitungsseminar hatte das Ziel, den Freiwilligen zu vermitteln, „dass man nicht alleine und mit nichts nach Uganda geht, sondern ganz reich und gefüllt, weil alles wichtige hat man in sich und das trägt man mit nach Uganda“. Das war die Botschaft, die der Seminarleiter vermitteln wollte. Dieses Öffnungsritual kann als Versuch verstanden werden, sich selbst zu öffnen und damit für „Neues“ offen zu sein. Das Erlebte soll dann nicht mehr in vorgefertigte Orientierungsraster eingeordnet werden, sondern die Öffnung ist eine Einladung, sich irritieren zu lassen. Konzepte der Diversity können dabei helfen, die Komplexität der Differenzen anzunehmen und sichtbar zu machen. Dabei geht es nicht darum zu bewerten, welche Differenzziehungen richtig oder falsch sind, sondern vielmehr dazu anzuregen, die komplexen Differenzen auszuhalten. Der Diversity-Ansatz ${ }^{6}$ macht die Bearbeitung von bipolaren Konstruktionen möglich, zu denen die jungen Erwachsenen neigen. Hierbei kommt die Ambiguitätstoleranz ins Spiel. „Sie

6 In Diversity-Ansätzen geht es um die Wertschätzung von Vielfalt und Heterogenität und somit kann dies als Gegenkonzept zur Diskriminierung und Homogenisierung verstanden werden. 
meint die Fähigkeit, sich auf Menschen und Situationen offen einzulassen, sie zu erkunden, sie nicht nach einem ,Alles-oder-nichts'-Prinzip als nur gut oder nur böse zu beurteilen. Es geht also um die Überwindung des 'Eindeutigkeitszwanges' und die Ermöglichung von neugieriger Exploration von Realitätsschichten, die einer verkürzenden instrumentellen Logik unzugänglich sind“ (Keupp 1994: 346).

Gerade die Mitarbeitenden in Entsendorganisationen stehen vor der Herausforderung, ihre eigene Konstruktionen und Deutungen stets zu reflektieren, ihre Gewordenheit zu hinterfragen und sich von bipolaren Deutungen zu distanzieren. Außerdem macht der Prozess der Begleitung es erforderlich, sich auf die Irritationen, Deutungen und Fragen der jungen Menschen einzulassen. Professionelle in der Begleitung von Freiwilligen müssen sich also in doppelter Weise auf Lebenswelten einlassen - erstens auf die Lebenswelten und Erlebnisse im Einsatzland und damit verbunden der Adressat_innen der jeweiligen Projekte und zweitens auf die Lebenswelt und Deutungen der jungen Menschen, die sie im internationalen Freiwilligendienst begleiten.

\subsection{Inbetweenness als Reflexionsmöglichkeit: Ambivalenzen aushalten}

Das zu Beginn als Ambivalenz und Nicht-Wissen-Wie gekennzeichnete Handeln der jungen Menschen kann für diese Öffnung und Reflexion als produktiver Raum genutzt werden. Diese Ambivalenz ermöglicht erst ein Nachdenken und Nachfühlen, welche von den Begleitenden nicht als Handlungsunfähigkeit oder Handlungsunsicherheit behoben werden muss, sondern in ihrem Potential der Reflexion gesehen werden kann. In der eben skizzierten Öffnung werden neue Deutungen möglich und das Erlebte wird möglicherweise nicht nur in vorhandene Deutungsmuster eingeordnet, sondern diese Deutungsmuster können von den neuen Erfahrungen irritiert werden. Die jungen Menschen greifen in ihrem alltäglichen Handeln häufig auf die bisherigen Deutungsmuster zurück. Die damit verbunden bipolaren Konstruktionen bieten ihnen Sicherheit, die ihnen auch nicht genommen werden darf. Dennoch kann meines Erachtens eine sozialpädagogische Begleitung genau hier ansetzen und zu Lern- oder Transformationsprozessen führen, indem sie das Ringen und Zweifeln der jungen Menschen in Form von Supervisionsrunden o. ä. aufgreift und sich mit den jeweiligen Ambivalenzen detailliert auseinandersetzt und keine vorschnellen Handlungszwänge und -praktiken anvisiert. Hier sei nochmals auf das Sozialpädagogische der Begleitung von Freiwilligen verwiesen: Sozialpädagogische Professionalität ist gekennzeichnet von Handeln in Paradoxien (Schütze 1992) - und somit Ambivalenzsituationen. In einer sozialpädagogischen Begleitung können die Ambivalenzen des Inbetweenness reflektiert werden, ohne vorschnell einseitig aufgelöst zu werden. 
Anknüpfend an Abschnitt 4.1 kann dabei insbesondere nochmals das Jugendalter aufgegriffen werden und die Tatsache, dass der Internationale Freiwilligendienst mehr als Arbeit ist. Zwar brauchen die jungen Menschen Orientierung und auch in ihren eigenen bipolaren Konstruktionen ist das Verlangen nach Sicherheit erkennbar, aber genau dies macht deutlich, dass sie begleitet oder motiviert werden müssen, Offenheit auszuhalten, andere Deutungen zuzulassen und sich von dem Erlebten irritieren zu lassen. Daher geht es weniger um klare Regeln, als vielmehr um die Bereitstellung von Reflexionsräumen, wie sie im Inbetweenness angelegt sind. Das Inbetweenness bietet außerdem die Möglichkeit eines geschützten Raumes, in dem Stereotype geäußert und bearbeitet werden können ohne dass sie sich dabei verfestigen müssen. Hierbei kann anstelle von statischen Deutungen kontextualisiertes Wissen erzeugt werden. Umso verwunderlicher erscheinen dabei Regelungen für zukünftige Freiwillige, wie sie mir bei Informationstagen großer Träger begegnet sind: Kein Alkohol trinken, nicht rauchen, in die Kirche gehen, am besten kein Handy, eine bestimmte Kleiderordnung einhalten. Ich möchte damit nicht sagen, dass mit diesen Themen keine Auseinandersetzung stattfinden soll, aber sie scheinen mir als statische Regelung an der Lebensrealität von jungen Menschen im internationalen Freiwilligendienst vorbeizugehen und nichts mit dem zu tun zu haben, was ich im Feld beobachten konnte - gerade weil der Internationale Freiwilligendienst mehr ist als Arbeit. Ähnlich wie im skizzierten Fall „Handeln nach klaren Prinzipien“ (s. o.) muss meines Erachtens eine reflexive Auseinandersetzung mit den Themen wie Armut, Alkoholkonsum, Religiosität usw. stattfinden. Die klaren Regelungen produzieren möglicherweise - in Bereichen, in denen sie angenommen werden - Handlungssicherheit und damit die Reduktion von Unsicherheit, führen aber auch dazu, dass keine weitere Auseinandersetzung stattfindet. Dabei sind Lernerfolge insbesondere dann zu verzeichnen, wenn kontextualisiertes Wissen erzeugt und angebracht werden kann. Damit ist gemeint, dass die jeweilige Handlungen und dass dem zugrundeliegende Wissen dem jeweiligem Kontext angemessen ist. Olivier (2013) nennt diese Form des Wissens transnationales Wissen und macht deutlich, dass die jeweils Handelnden unterscheiden, wie sie in welchem Nationalkontext agieren. Regelungen, die als solche von den jungen Menschen nicht angenommen werden, weil sie der Lebensrealität von jungen Menschen - auch im neuen Kontext - widersprechen, erzeugen anstelle von Sicherheit eher ein Gefühl von Unrecht. Beispielsweise steht der Idee eines Handyverbotes die empirische Realität gegenüber, dass auch die meisten jungen Menschen in Uganda mit ähnlichem Bildungsstatus der Freiwilligen ein Handy besitzen und die Kommunikation sowohl zwischen den Freiwilligen, als auch zwischen Freiwilligen und ugandischen Freund_innen und zwischen Freiwilligen und Freund_innen und Familie in Deutschland oder in anderen Ländern über das Medium Handy stattfindet. Die Angst, dass die jungen Menschen nicht im Einsatzland ankommen, 
wenn sie mit ihrem Herkunftsland in regem Kontakt stehen, ist meines Erachtens erstens aus Perspektive der Transnationalitätsforschung (bspw. Pries 1997) nicht gegeben, da sich das Ankommen und mit dem Bisherigen in Kontakt stehen, nicht widersprechen müssen. Vielmehr ist in Forschungen die Gleichzeitigkeit von transnationalen Kontakten und regionalen Kontakten erkennbar (Mau 2007). Zweitens geht es auch hier viel mehr darum, die jungen Menschen bei einem reflexiven Umgang zu begleiten, möglicherweise auf Zusammenhänge hinzudeuten, wenn es zu Schwierigkeiten des Ankommens kommt, aber nicht generelle Handlungsregeln aufzustellen: Wann ist welches Verhalten angebracht? Welche Bilder produziere ich mit meinem eigenen Handeln?

Das Inbetweenness lädt zur Reflexion und zur Auseinandersetzung mit diesen Fragen ein. Die jungen Menschen wissen nicht, wie sie sich verhalten sollen und ringen um angemessenes Verhalten. Somit ist nach einem Öffnungsprozess - wie er möglicherweise auf einem Vorbereitungsseminar angelegt ist - insbesondere von einer Begleitung im Sinne einer Supervision oder eine kollegialen Beratung auszugehen. Wer aber kann solche Reflexionsprozesse anleiten und wie könnte dies aussehen? Auf diese Frage soll im Folgenden eingegangen werden.

\subsection{Vorhandene Netzwerke nutzen}

$\mathrm{Ob}$ über Kommunikationsmedien wie Handy und Internet oder in Face-to-FaceInteraktionen: Es entstehen Beziehungen zwischen den Freiwilligen und auch über die Freiwilligengruppe hinaus. Im empirischen Material lassen sich drei zentrale Gesellungsformen nachzeichnen: (1) die (transnationale) Freiwilligengruppe, (2) Gesellung über Generationen hinweg, also beispielsweise Beziehungen zu europäischen Entwicklungshelfer_innen und (3) Gesellungen von angeblich Gleichen, die über die Zugehörigkeitsform „beide Welten kennen“ konstruiert sind (vgl. ausführlich Mangold 2012a). Letzteres sind somit auch ugandische Staatsbürger_ innen, die aber eine begrenzte Zeit ihres Lebens in Europa oder Deutschland verbracht haben. Diese Personen werden als „Vermittlungspersonen“ bezeichnet. Im Folgenden sollen zwei Aspekte hervorgehoben werden, welche für die Begleitung von Freiwilligen unterstützend sein können:

\subsection{Vermittlungspersonen einbeziehen}

Als empirisches Beispiel für eine solche Vermittlungsperson kann Emanuel herangezogen werden: Emanuel, ugandischer Staatsbürger, ist 29 Jahre alt, hat vier Jahre in Deutschland Sozialpädagogik studiert, kam dann zurück nach Uganda, wo er nun in einem sozialen Projekt arbeitet und für die Freiwilligen aus Deutschland zuständig ist. Er wohnt auch mit einigen Freiwilligen zusammen in einer großen Wohngemeinschaft. An einem Filmabend wird er gezielt als „Vermittler“ 
nachgefragt. Der Spielfilm „Der letzte König von Schottland“7 vermittelt den Zuschauenden die Geschichte des Landes und diese Auseinandersetzung mit der Geschichte wird gezielt mit Emanuel gesucht, so die Annahme. Emanuel ist als Repräsentant von Uganda anwesend, als Betroffener, der jedoch selbst die Zeit von Idi Amin nicht miterlebt hat, da er erst danach geboren wurde. In dieser Rolle wird er nach dem Film auch von Irene gefragt, „ob er denkt, dass das die Realität wiedergibt“. Es scheint also von Bedeutung zu sein, den Spielfilm mit der Realität abzugleichen. Dazu wird aktiv ein „Ugander“ gefragt. Emanuel berichtet daraufhin als „Ugander" und als Mensch, der aufgrund seines Alters zwar nicht direkt von den Geschehnissen unter dem Diktator Amin betroffen war, aber dessen Familiengeschichte von dieser Zeit geprägt ist.

Beim Filmschauen werden Grashüpfer mit Tomaten-Dip geknabbert. Grashüpfer gelten im Dezember in Uganda als Leckerei, die mit viel Aufwand gefangen und gerne verspeist werden. Meist isst man sie frittiert als eine Art Knabberei. Was für die Freiwilligen als Exotismus erlebt wird, wird von Emanuel mit Selbstverständlichkeit getan. Aber auch Irene entwickelt selbstverständliche Routinen im Essen von Grashüpfern und sagt, dass „der Kopf am besten [sei]“. Zu den Grashüpfern gibt es Tomatendip - was in Uganda gewöhnlich nicht zu Grashüpfern serviert wird - und beides wird schließlich von Emanuel „in zwei Schüsseln" aufgeteilt, „so dass auf jedem Sofa etwas zu 'knabbern' steht". Dies erinnert an Chipstüten, die man in einer Runde bei einem DVD-Abend in zwei Gefäße verteilt, so dass alle geschickt nehmen können. Der Dip - welcher als „westliche“ Art verstanden werden kann (multikulturelle Form des Knabberns) - ist ein Zeichen für eine Transformation der ugandischen Praxis des Grashüpferessens und kann somit als „transnationale Form“ des Essens von Grashüpfern begriffen werden.

Hierbei scheint es sich also um ein Experimentierfeld für die Freiwilligen zu handeln, das durch die Anleitung und Vermittlungsposition von Emanuel mitgestaltet ist. Die Gesellungsform rund um „Vermittlungspersonen“ ermöglicht also eine spezifische Form des Ausprobierens und Experiementierens. Emanuel ist eine Person, die sowohl die Lebenswelt in Deutschland kennt, als auch Uganda und die Geschichte des Landes. Er kennt beides und nutzt dieses Wissen in seinen alltäglichen Gesprächen und Handlungen. Emanuel kann somit als „Vermittlungsperson“ zwischen „hier" und „dort" verstanden werden. Vermittlung findet dabei nicht nur im Vormachen (z. B. Grashüpfer essen) oder Diskutieren und Erläutern (z. B. Film schauen) statt, sondern ist in das (alltägliche) Handeln und Denken der „Vermittlungspersonen“ eingelagert, indem „,hier“ und „dort“ aufeinander bezogen werden und somit Vermittlung nicht nur explizit sondern auch implizit stattfindet.

7 Ein Politthriller, der an tatsächliche Ereignisse aus der Regierungszeit des ugandischen Diktators Idi Amin anknüpft. 
So stellt sich beispielsweise die Frage, warum Zwischenseminare meist von Menschen aus Deutschland durchgeführt werden und nicht vermehrt auf das Wissen der Menschen vor Ort gesetzt wird, die bereits in Deutschland waren? Die enge Zusammenarbeit mit den Partnerorganisationen ist hier meines Erachtens der Schlüssel zum Erfolg. Dabei geht es nicht nur darum eine gute Kooperation zu gestalten, sondern darum eine gemeinsame Idee zu entwickeln. Beispielsweise weltwärts darf dann nicht mehr ein nationales Projekt bleiben (auch wenn die Gelder vom deutschen Staat kommen), sondern muss als transnationales Projekt verstanden werden. So sollte auch die sozialpädagogische Begleitung einerseits darauf achten, sowohl aus Perspektive der Organisation Menschen zu vermitteln, welche die jungen Menschen zur Reflexion ihrer Differenzkonstruktionen anhand von eigener Erfahrungen im Ausland unterstützen können, andererseits auch systematisch Menschen im Einsatzland in die Begleitung mit einzubeziehen. Dabei geht es nicht darum, die „Vermittlungsleistung“ zu übernehmen und neue Deutungen und Sichtweisen zu erlernen, sondern in der gemeinsamen Reflexion neue Deutungen zu ermöglichen und Ambivalenzen nicht vorschnell auflösen zu müssen.

\subsection{Peergruppen der Freiwilligen stärken - insbesondere Zweierkonstellationen wirken unterstützend}

Als weitere Form der Unterstützung kann die Freiwilligengruppe selbst herausgestellt werden. Ob gewollt oder nicht, die Freiwilligen erleben sich als Gruppe und unterstützen sich dabei bei vielfältigen alltäglichen Herausforderungen, aber auch in Krisen und Ängsten. Dabei lässt sich insbesondere die Zweierbeziehung unter Freiwilligen herausstellen. „Zweisamkeit“ wird hierbei vor allem in homogenen Paarkonstellationen beobachtet und als sich aufeinander verlassen können erlebt. Die „beiden“ sind füreinander da und zwar in einer verbindlicheren Art und Weise, wie es eine Gruppe gewährleisten kann. Dabei werden sowohl Alltäglichkeiten miteinander geteilt, als auch insbesondere bei Schwierigkeiten und Beratungsbedarf aufeinander eingegangen.

Daher ist es für die gegenseitige Unterstützung und die informellen Unterstützungsprozesse unter Freiwilligen sinnvoll, Freiwillige nicht allein in eine Region zu entsenden, sondern zumindest als Zweierkonstellation. Das muss nicht bedeuten, dass diese Freiwilligen in der gleichen Einrichtung arbeiten oder zusammen wohnen müssen, aber dass die Möglichkeit besteht füreinander auf einfachem Wege erreichbar zu sein. Dies mag Bedenken auslösen in Bezug auf Gruppenbildung und Nicht-Ankommen in der Gesellschaft des Einsatzlandes. Dem sei entgegengehalten, dass die Freiwilligencommunity auch ohne Zweierkonstellationen entsteht und die jungen Menschen sich vernetzen, was nicht mit einem Nicht-Ankommen in dem Einsatzland verbunden sein muss. Aber die Möglichkeit für eine „verlässliche" Zweierkonstellation erscheint diesen Rekonstruktionen zufolge für die Unterstützung der Freiwilligen produktiv. 


\section{Fazit}

Abschließend kann festgehalten werden, dass bisherige Begleitkonzepte weiter ausgebaut werden und Eigeninitiativen der Freiwilligen in der Begleitung aufgegriffen werden können. Hierbei sei auch auf vielfältige Initiativen und Veröffentlichungen von Freiwilligen selbst verwiesen, welche sich (selbst)reflexiv mit dem eigenen Freiwilligendienst auseinandersetzen (bspw. Stark/Weber 2012; Schmitz 2007; VENRO 2009). Es geht also nicht darum, das Rad neu zu erfinden, sondern an dem Bisherigen weiterzumachen und bestehende Modelle der Begleitung selbstkritisch zu modifizieren und weiterzuentwickeln. Dabei sei sowohl an die inhaltliche als auch an die zeitlich-strukturelle Ausgestaltung gedacht. Die dafür relevanten Ergebnisse der empirischen Analyse werden hier nochmals überblickartig gebündelt:

- Freiwilligendienst ist mehr als Arbeit: So sollte der Alltag der jungen Menschen zum Thema gemacht werden, ohne sie dabei in ihrem jungen Erwachsen-Sein zu gefährden. Auch jugendspezifische Fragen werden im Alltag verhandelt.

- Diversity: Bereits in der Anleitung und Vorbereitung der Freiwilligen sollte auf Vielfalt und Unterschiedlichkeit geachtet werden und eine kritische Haltung zu statischen Kategorisierungen wie „Rasse“, Alter, Geschlecht usw. eingenommen werden. Hierzu bieten vor allem die Ansätze der diversity education Möglichkeiten eines kritischen Umgangs.

- Inbetweenness als Reflexionsmöglichkeit: In den Verortungen der jungen Menschen lässt sich ein Ringen erkennen, welches auf eine Offenheit und ein Suchen hindeutet. Auch der Schritt ins Ausland ist als Offenheit und möglicherweise auch als Neugierde zu verstehen. Diese Offenheit sollte weiter genutzt werden, die Neugier nicht durch bipolare Deutungen eingedämmt werden. Dass sich dabei auch Unsicherheiten und Ängste einschleichen und der Rückgriff auf bipolare Deutungen zu schnellerem Erfolg und mehr Sicherheit führt, kann dabei nicht überraschen. Jedoch liegt genau hierin der Bildungsauftrag. Dieser Prozess kann nur als Selbstbildungsprozess ablaufen, braucht aber zumindest Irritation von außen bzw. Motivation im Inbetweenness zu verweilen und Ambivalentes auszuhalten.

- Vorhandene Netzwerke nutzen: Bei diesen Reflexionsprozessen können vorhandene Netzwerke genutzt werden und gezielt die Menschen einbezogen werden, die „beide Welten kennen“ und die das Inbetweenness aushalten können. Aber auch die Freiwilligen selbst sollten in ihrer gegenseitigen Unterstützungsleistung gestärkt werden und Peer-Beratungsprozesse angeregt und genutzt werden.

Die bereits bestehenden Konzepte zur pädagogischen Arbeit in der Begleitung von Freiwilligen (bspw. GIZ 2014) können einerseits durch die Analyseergebnisse unterstrichen werden, andererseits auch erweitert werden. Es konnte insbesondere 
darauf aufmerksam gemacht werden, dass die jungen Menschen während der Zeit des Freiwilligendienstes in sogenannten Inbetween-Situationen offen für Reflexion und Auseinandersetzung sind und dass es lohnenswert und notwendig ist, an den alltäglichen Ambivalenzerfahrungen mit den jungen Menschen zu arbeiten. Wenn es also beim internationalen Freiwilligendienst wirklich darum gehen soll, den Blick der jungen Menschen zu öffnen und die Gefahr der Stereotypenverfestigung einzudämmen, dann führt kein Weg an einem intensiven Reflexionsprozess und einer Begleitung während des Freiwilligendienstes vorbei. Dabei soll hier nochmals explizit betont werden, dass die Verarbeitung dieser Erfahrungen nicht nur in angeleiteten Supervisionen oder Workshops stattfindet, ganz im Gegenteil, die jungen Menschen unterstützen sich informell - meines Erachtens sind hierbei aber Inputs von außen notwendig, um nicht gemeinsam Stereotype zu verfestigen, sondern die Perspektiven und Deutungen in einem gemeinsamen Lernprozess zu erweitern. Hierfür reichen Ansprechspartner_innen für Krisensituationen vor Ort nicht aus, sondern es bedarf einer kontinuierlichen - eben gerade nicht nur an artikulierte Krisen geknüpfte - Form der Reflexion, wie es beispielsweise durch Supervisionsgruppen zu gewährleisten wäre. Dabei könnten die sogenannten Vermittlungspersonen und auch die Freiwilligencommunity als kollegiale Berater_innen mit einbezogen werden. Hier werden nicht nur Herausforderungen im Arbeitsleben zu reflektieren sein, sondern das Alltagsleben und die damit verbundenen Statusfragen und -herausforderungen werden zum Teil des Supervisionsprozesses. Für die Ausgestaltung der Vor- und Nachbereitung lässt sich folgendes festhalten: Die Vorbereitung stellt insbesondere die Möglichkeit dar, den jungen Menschen (und deren Familien) Unsicherheit zu nehmen und Fragen zu beantworten. Im Idealfall finden hier bereits Öffnungsprozesse statt, wie wir sie am Beispiel von Lenas Erfahrungen beobachten konnten. Ansonsten sollte hier vermieden werden statische Bilder des Einreiselandes zu zeichnen und Stereotype zu verstärken. Die Rückkehrarbeit steht vor der Herausforderung die Erfahrungen in das Leben nach dem Freiwilligendienst zu transformieren. Das Leben nach dem Freiwilligendienst ist jedoch meist erneut von Veränderungen gekennzeichnet (z. B. Beginn des Studiums), so dass wenig Zeit für eine ausführliche Reflexion bleibt. Um die Erfahrung des Freiwilligendienstes lebendig zu halten, suchen sich die jungen Menschen jedoch meist andere ehemalige Freiwillige, mit denen sie die gemeinsamen Erlebnisse austauschen können.

Abschließend, um hier nicht missverstanden zu werden: Es bedarf keiner Rundum-Betreuung im internationalen Freiwilligendienst. Meine empirische Analyse zeigt, dass die jungen Menschen handlungsfähig sind und in der Lage sind, sich bei Schwierigkeiten und Herausforderungen Unterstützung zu organisieren. Der für die jungen Menschen bereitgestellte Raum der Orientierung oder der Auszeit darf nicht durch die Begleitung überlagert werden. Die Begleitung sollte daher vor allem das aufnehmen, was von den jungen Menschen selbst zum Thema gemacht 
wird und dabei insbesondere im Sinne von Diversity vielfältige Deutungen und somit Öffnungen ermöglichen. Dabei kann das „Ringen um angemessenes Verhalten" zum Potential der Reflexion werden und im Inbetweenness stereotypen Deutungen bearbeitet werden.

\section{Literatur}

Beck, Ulrich (1993): Die Erfindung des Politischen, Frankfurt am Main.

BMZ (2007): weltwärts. Der Freiwilligendienst des Bundesministeriums für wirtschaftliche Zusammenarbeit und Entwicklung. Richtlinie zur Umsetzung des entwicklungspolitischen Freiwilligendienstes „weltwärts“, Bonn.

Broek van der, Lida (1993): Am Ende der Weißheit, Vorurteile überwinden, Ein Handbuch, Berlin.

Bührmann, Thorsten und Irmgard Rehaag (2009): Erleben des Fremden und Reflektieren des Eigenen. Pädagogische Überlegungen zu Auslandsaufenthalten im Studium, in: der pädagogische Blick. Zeitschrift für Wissenschaft und Praxis in pädagogischen Berufen, 17. Jg. , Heft 1,2009 , S. 35-47.

Fischer, Jörn (2011): Freiwilligendienste und ihre Wirkung - vom Nutzen des Engagements, in: Aus Politik und Zeigeschichte (APuZ), Heft 48/2011, S. 54-62.

Fürstenau, Sara und Heike Niedrig (2007): Hybride Identitäten? Selbstverortungen jugendlicher TransmigrantInnen, in: Diskurs Kindheits- und Jugendforschung 2 (2007) 3, S. 247-262.

Gaiser, Wolfgang und Johann de Rijke (2001): Gesellschaftlich Beteiligung der Jugend. Handlungsfelder, Entwicklungstendenzen, Hintergründe, in: Aus Politik und Zeitgeschichte, B44/2001, S. 8-16.

GIZ (2014): weltwärts mit der GIZ, Pädagogische Dokumentation, Bonn.

Glaser, Barney G. und Anselm Strauss (1998): Grounded Theory. Strategien qualitative Forschung, Bern.

Goel, Urmila (2011): Rassismus- und privilegienkritische Bildungsarbeit, In: weltwärts pädagogisch begleiten, Erfahrungen aus der Arbeit mit Freiwilligen und Anregungen durch die Fachtagung in Bonn (18.-20. April 2010), Kassel, S. 24-31, hrsg. von Diana Grundmann und Bernd Overwien, http://www.uni-kassel.de/upress/online/frei/978-3-86219198-7.volltext.frei.pdf (Zugriff am 30.09.2011)

Günther, Marga (2009): Adoleszenz und Migration. Adoleszenzverläufe weiblicher und männlicher Bildungsmigranten aus Westafrika, Wiesbaden.

Haas, Benjamin (2012): Ambivalenz der Gegenseitigkeit - Reziprozitätsformen des weltwärtsFreiwilligendienstes im Spiegel der Postkolonialen Theorie, Köln.

Hudson, Sheena und Kerr Inkson (2006): Volunteer overseas development workers: the hero's adventure and personal transformation, Career Development International, Vol. 11 Iss: 4, S. 304-320.

Jones, Andrew (2005): Assessing international youth service programmes in two low income countries, in: Voluntary Action: The Journal of the Institute for Volunteering Research 7 (2), S. 87-100.

Keupp, Heiner (1994): Ambivalenz postmoderner Identität, in: Riskante Freiheiten, Individualisierung in modernen Gesellschaften, Frankfurt am Main, hrsg. von Ulrich Beck und Elisabeth Beck-Gernsheim. 
King, Vera (2005): Adoleszenz und Migration - eine verdoppelte Transformationsanforderung. Am Beispiel des Bildungserfolgs als Thema der adoleszenten Generationenbeziehungen in Familien mit Migrationshintergrund, in: Kindheit jenseits von Trauma und Fremdheit. Psychoanalytische Erkundung von Migrationsschicksalen im Kinder- und Jugendalter, hrsg. von Peter Bründl und Ilany Kogan. Frankfurt am Main. S. 30-51.

Klocke, Karla (2013): „Hilfe zur Selbsthilfe“ (nur) angenommen?! WELTWÄRTS aus Sicht der Partnerorganisationen, unveröffentlichte Bachelor-Arbeit, Universität Hildesheim.

Leiprecht, Rudolf und Dita Vogel (2008): Transkulturalität und Transnationalität als Herausforderung für die Gestaltung Sozialer Arbeit und sozialer Dienste vor Ort, in: Soziale Arbeit und Transnationalität. Herausforderungen eines spannungsreichen Bezugs, hrsg. von HansGünther Homfeldt, Wolfgang Schröer und Cornelia Schweppe, Weinheim- München, S. 25-44.

Lough, Benjamin J. (2013): Social Work Perspectives on International Volunteer Service, in: British Journal of Social Work (2013), p. 1-16.

Mangold, Katharina (2011): Herausforderungen im jungen Erwachsenenalter - junge Menschen im Freiwilligendienst, in: weltwärts pädagogisch begleiten. Erfahrungen aus der Arbeit mit Freiwilligen und Anregungen durch die Fachtagung in Bonn (18.-20. April 2011), Kassel, hrsg. von Diana Grundmann und Bernd Overwien, S. 20 - 24, http://www.uni-kassel.de/ upress/online/frei/978-3-86219-198-7.volltext.frei.pdf (Zugriff am 12.9.2013).

Mangold, Katharina (2012a): Beide Welten kennen - Gesellungsformen junger Erwachsener im Internationalen Freiwilligendienst, in: Transmigration und Soziale Arbeit, hrsg. Von Andreas Herz und Claudia Olivier, Weinheim- München, S. 185-205.

Mangold, Katharina (2012b): 'Struggling to behave appropriately' - Challenges During International Volunteering, in: Third World Quarterly 33 [8] 2012, Special Issue: The Personal and the Professional in Aid Work, S. 1493-1509.

Mangold, Katharina (2013): Inbetweenness: Jugend und transnationale Erfahrungen, Weinheim und München.

Mau, Steffen (2007): Transnationale Vergesellschaftung, Frankfurt am Main.

Müller, Burkhard (2006): Sozialpädagogisches Können. Ein Lehrbuch zur multiperspektivischen Fallarbeit, 4. Aufl, Freiburg.

Mundorf, Margret (2000): Christliche Freiwilligendienste im Ausland. Lernprozesse und Auswirkungen auf die Lebensentwürfe junger Menschen, Münster-New York-München-Berlin.

Olivier, Claudia (2013): Brain Gain oder Brain Clash? Implizites transnationales Wissen im Kontext von Rückkehr-Migration, in: Transnationales Wissen und Soziale Arbeit, hrsg. von Anne Duscha, Desiree Bender, Lena Huber und Kathrin Klein-Zimmer, Weinheim-München, S.181-205.

Pries, Ludger (1997): Neue Migration im transnationalen Raum, in: Transnationale Migration. Soziale Welt, Sonderband 12, Baden-Baden. S. 15-44.

Schmitz, Charlotte (2007) (Hrsg.): Lernen und tatkräftiges Helfen. Der BMZ-Freiwilligendienst „weltwärts, ein Dossier von Arbeitskreis Leben und Helfen in Übersee e.V. in Zusammenarbeit mit der Redaktion „welt-sichten“.

Schütze, Fritz (1992): Sozialarbeit als „bescheidene“ Profession, in: Erziehung als Profession: zur Logik professionellen Handelns in pädagogischen Feldern, hrsg. von Bernd Dewe, Wilfried Ferchhoff und Frank-Olaf Radtke, Opladen. S. 132-170. 
Schwinge, Brigitte (2011): Verkehrte Welten: Über die Umkehrung der Verhältnisse von Geben und Nehmen. Der weltwärts-Freiwilligendienst als Selbstbehandlung im Kulturkontakt zwischen Deutschland und Südafrika. Bonn-Manama-New York-Florianópolis.

Simpson, Kate (2004): 'Doing development': the gap year, volunteer-tourists and a popular practice of development. In: Journal of International Development, 16: 681-692, in Wiley InterScience (www.interscience.wiley.com), DOI: 10.1002/jid.1120.

Stark, Annika und Weber, Julian (2012): „Ehrt Euer chlorfreies Trinkwasser“. Eine kritische Auseinandersetzung mit weltwärts-Blogs, in: „Wer anderen einen Brunnen gräbt“. Rassismuskritik/Empowerment/Globaler Kontext, Broschüre hrsg. von BER (Berliner Entwicklungspolitischer Ratschlag).

Stauber, Barbara und Walther, Andreas (2002): Junge Erwachsene, in: Handbuch Kinder- und Jugendhilfe, hsrg. von Wolfgang Schröer, Norbert Struck und Mechthild Wolff, WeinheimMünchen, S. 113-143.

Steinkühler, Sandra (2014): Internationale Freiwilligendienste Untersuchung zum Einfluss der Auswahlverfahren der Entsendeorganisationen auf die soziodemographische Teilnehmerstruktur von Internationalen Freiwilligendiensten, unveröffentlichte Bachelor-Arbeit, Universität Hildesheim.

Stern, Tobias und Scheller, Jan-Oliver (2011): Der entwicklungspolitische Freiwilligendienst weltwärts, Evaluierungsbericht 056, Bonn: Bundesministerium für wirtschaftliche Zusammenarbeit und Entwicklung, www.bmz.de/de/publikationen/reihen/index. html\#evaluierungen (Zugriff am 20. Februar 2012).

Töpfl, Florian (2008): Egotrip ins Elend, in: Süddeutsche Zeitung Magazin, Heft 19/2008, http:// sz-magazin.sueddeutsche.de/texte/ anzeigen/24384/ (Zugriff am 20.09.11)

VENRO (2009): Going beyond weltwärts. Ansätze für die erfolgreiche Integration des Globalen Lernens und eines Reversprogrammes in entwicklungspolitischen Freiwilligendiensten, Diskussionspapier 2, Bonn, www.venro.org/fileadmin/redaktion/dokumente/ag_bildung/Dezember_2009/Weltwaerts_v04_WEB.pdf, (Zugriff am 5.9.2012) 